\title{
Pathomechanics of Syndesmotic Injuries
}

\author{
Pilar Martínez de Albornoz ${ }^{1}$, Manuel Monteagudo
}

\begin{abstract}
Syndesmotic injuries have gained interest in recent years since they are present in $13 \%$ of ankle fractures and up to $20 \%$ of ankle sprains. The clinical suspicion of these injuries should lead the surgeon to ask for 3D-computed tomography images to avoid misdiagnosed injuries that may change management planning. The concept of dynamic joint stabilization is important, and overall knowledge of anatomy, biomechanics of a healthy and pathological syndesmosis will provide an objective starting point to improve diagnosis, mechanical repair, and rehabilitation. This review highlights basic and advanced biomechanical concepts, an update of the scientific evidence, and laboratory results with clinical outcomes for a better comprehension of treatment options.
\end{abstract}

Keywords: Ankle fractures, Biomechanics, Foot and ankle, Syndesmosis.

Journal of Foot and Ankle Surgery (Asia Pacific) (2021): 10.5005/jp-journals-10040-1194

\section{INTRODUCTION}

The syndesmosis joint provides stability during weight-bearing in both the coronal and axial planes and provides flexibility to the ankle mortise while walking on uneven ground. It has the function of acting as a dynamic stabilizer of the ankle joint complex. The concept of a dynamic joint stabilizer is important, and overall knowledge of the biomechanics of a healthy and pathological syndesmosis will provide an objective starting point to improve diagnosis, treatment, and rehabilitation.

The importance of the syndesmosis is reflected by figures of injuries. Almost $13 \%$ of ankle fractures ${ }^{1}$ and between $18 \%$ and $20 \%$ of ankle sprains diagnoses ${ }^{2}$ are associated with syndesmotic injuries. Injuries to the syndesmotic ligaments require longer immobilization and recovery times. ${ }^{3}$ It is therefore important to suspect syndesmosis injury to ensure an early diagnosis and appropriate and timely treatment, given that a missed diagnosis or delayed treatment can have serious consequences for the ankle joint.

For these reasons, a growing interest in this topic in recent years has led to an exponential rise in the number of publications that address the topic: 61 mean annual publications from 1956 to 1972, 347 mean annual publications from 1973 to 2008, and 1,747 mean annual publications from 2009 to $2020 .^{4}$

\section{Mechanical Anatomy}

Ankle ligaments together with joint bony congruence are the main stabilizers of the ankle. Injury to one or several of these structures can cause ankle instability which, in turn, produces chronic ankle pain and can also lead to secondary arthritis in this joint. Stability of the weight-bearing ankle and the syndesmosis can be attributed to:

- Distal osseous anatomical stability of the tibia and fibula: "incisura fibularis".

- Ligamentous stability is provided by three main ligaments: "lesser ring syndesmosis".

The ankle syndesmosis is a fibrous joint comprised of the distal end of the tibia and the fibula that fit perfectly in the cartilaginous joint region called the "incisura fibularis". The articular part of the tibia that contributes to the structure of the syndesmosis has a
1,2Department of Orthopaedic Surgery and Traumatology, Hospital Universitario Quironsalud Madrid, Pozuelo de Alarcon, Madrid, Spain

Corresponding Author: Pilar Martínez de Albornoz, Department of Orthopaedic Surgery and Traumatology, Hospital Universitario Quironsalud Madrid, Pozuelo de Alarcon, Madrid, Spain, Phone: +34600887262, e-mail: pilarmalbornoz@hotmail.com

How to cite this article: Martínez de Albornoz P, Monteagudo M. Pathomechanics of Syndesmotic Injuries. J Foot Ankle Surg (Asia Pacific) 2021;8(4):162-167.

Source of support: Nil

Conflict of interest: None

concave morphology with a rough surface, a distal base, and a vertex $6-8 \mathrm{~cm}$ from the tibiotalar joint. ${ }^{5}$ The anterolateral edge of the tibia forms the anterior tubercle, or Tillaux-Chaput's tubercle, while the posterolateral one forms Volkmann's tubercle. ${ }^{5}$ The fibular joint surface that faces the tibia presents a convex triangular shape, with an anterior tubercle called Wagstaffe-Le Fort and a posterior tubercle of less significance. The tibiofibular syndesmosis has some degree of movement. This movement depends on the position of the ankle and its adaptation to the movement of the talar dome that has a trapezoidal shape, broader in the anterior region and narrower at the posterior. During dorsiflexion of the ankle, the fibula moves upward and rotates externally, while in plantar flexion the fibula descends slightly and rotates internally (Figs 1 to 3 ).

The distal end of the tibia and the fibula are joined together by a strong ligamentous complex composed of four ligaments: the anterior inferior tibiofibular ligament (AITFL), the posterior inferior tibiofibular ligament (PITFL), the transverse tibiofibular ligament (TTFL), and the interosseous tibiofibular ligament (ITFL). There is a gradual anatomical transition from the intraosseous membrane (IOM) to the interosseous ligament (IOL), and even to the AITFL and PITFL. This ligamentous complex counteracts the rotational, axial, and translation forces the syndesmosis is subjected to.

\section{BIOMECHANICS}

The syndesmosis joint bears loads in the coronal and axial planes, of axial compression from heel strike and of rotation by 


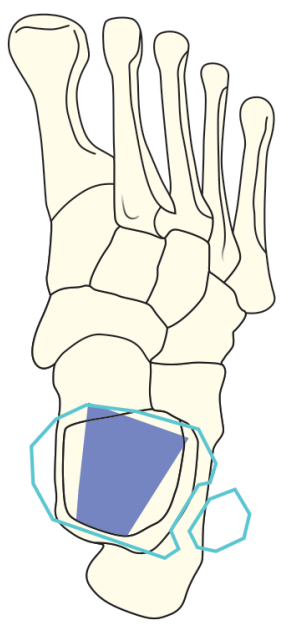

Fig. 1: Axial projection shows the relation between the talus-tibia-fibula

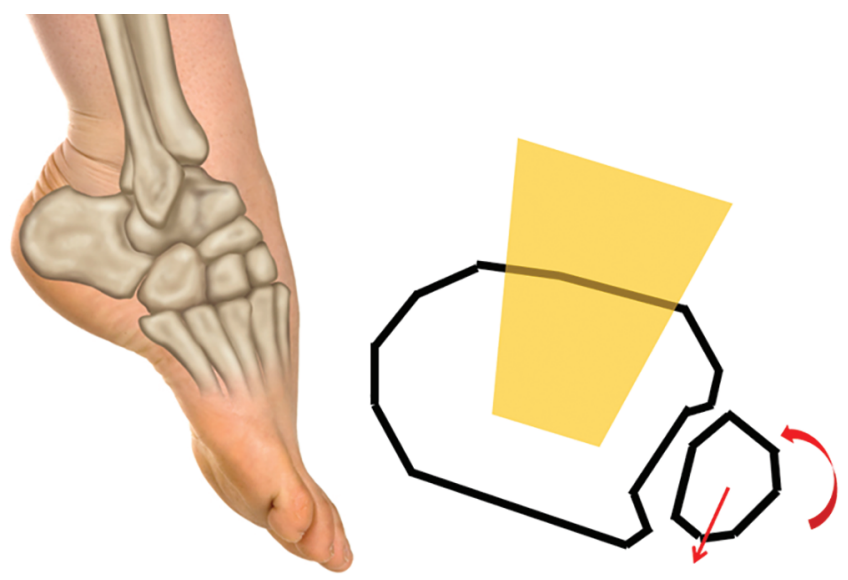

Fig. 3: During plantar flexion of the ankle, the fibula descends slightly and rotates internally

talar translation. These loads usually occur together, and the combination of the syndesmosis and the subtalar joint should be able to absorb the loading and impacts in all the planes. The syndesmosis is, therefore, a three-dimensional joint that bears moments of force in several planes. The structures involved are:

- In the coronal plane: the distal osseous structures of the tibia and the fibula in the incisura fibularis affect the close fit of the talocrural mortise when faced with axial forces. The IOL acts as a "spring" ligament, allowing a small separation between the medial and lateral malleolus during dorsiflexion of the talocrural joint, thus permitting the closest fitting of the talus into the mortise. In this way, the IOL functions as a shock absorber for the forces at the heel strike phase of gait (first rocker) and stabilizes the talocrural joint during full contact with the floor (second rocker). ${ }^{6}$

- In the axial plane: the three ligaments surrounding the syndesmosis (AITFL, PITFL, and ITFL) are responsible for maintaining a correct reduction of the incisura fibularis while adapting to rotational moments.

Most studies on syndesmotic biomechanics are carried out on cadavers that lead to a degree of bias. One such difference corresponds to the elasticity module and weight-bearing moments

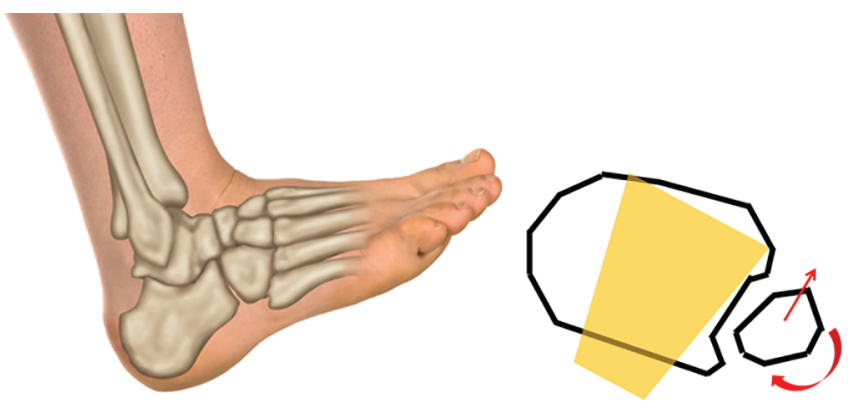

Fig. 2: During dorsiflexion of the ankle, the fibula moves upward and rotates externally

that can be applied around the joint. One of the biomechanical studies of greatest impact that focused on the relevance of each ligament in the syndesmosis was conducted by Ogilvie-Harris et al. ${ }^{7}$ These authors studied the resistance of each ligament to tibiofibular diastasis. It is important to bear in mind that in this study the tibiotalar joint of the specimens was disarticulated, and only an isolated lateral traction force was applied until separation of $2 \mathrm{~mm}$ was reached. The relative importance of each ligament for the joint's stability was found to be: $35 \%$ for the AITFL, 33\% for the transverse ligament, $22 \%$ for the IOL, and 9\% for the PITFL. ${ }^{7}$

Subsequently, further studies have been developed in which rotational and weight-bearing forces more similar to physiological forces were applied. ${ }^{8}$ Clanton et al. ${ }^{8}$ studied the relative contribution of the three most important ligaments of the syndesmosis (anterior, posterior, and interosseous) to rotational stability. The most frequent mechanism of injury was that caused by external rotation and a forced dorsiflexion moment of the ankle. The authors ${ }^{8}$ showed that the AITFL is responsible for $24 \%$ of syndesmotic resistance to this mechanism of injury, and the first to be damaged with this type of ankle movement. ${ }^{9}$ The superficial PITFL was an important stabilizing structure (15.1\%) when an internal rotational force was applied from the fibula. The same authors ${ }^{8}$ also studied the mobility of the syndesmosis in weight-bearing and non-weight-bearing scenarios. They concluded that mobility was extremely limited in all directions, including the rotational plane. The fibula was displaced $3.3 \mathrm{~mm}$ relative to the tibia in the sagittal plane (anterior-posterior). By applying external rotation, the posterior displacement of the fibula was $2.6 \mathrm{~mm}$ and, when an internal rotational force was applied the anterior displacement was $0.7 \mathrm{~mm}$. When each ligament of the syndesmosis was sectioned and an external rotational force applied, these articular ranges increased in the sagittal plane: translation of $9.4 \mathrm{~mm}$ after sectioning from posterior to anterior, and $10.2 \mathrm{~mm}$ translation while sectioning anterior to posterior. Therefore, injury to the syndesmotic ligaments increases rotation and translation of the fibula during weight-bearing and in external rotation. ${ }^{10}$ This results in an exponential increase in tibiotalar forces and affects the post-traumatic prognosis of the joint. Opening of the mortise by $1 \mathrm{~mm}$ reduces the tibiotalar contact area by $42 \%{ }^{11,12}$ and can lead to instability and the development of premature ankle arthritis.

\section{Syndesmotic Pathomechanics}

Syndesmosis injuries can occur with or without joint fractures. The most frequent syndesmotic injury is produced by external rotation and a moment of maximum dorsiflexion of the ankle. ${ }^{13}$ The injury can occur in any type of fracture but is more frequently associated with those produced with the ankle in pronation-external 
rotation (PER), supination-external rotation (SER), or in proximal fractures of the fibula (including the Maisonneuve fracture-a spiral fracture of the proximal third of the fibula-and Dupuytrentransverse fracture-associated with injury of the distal tibiofibular syndesmosis). Figures 4 to 11 describe the sequence of ligamentous and osseous lesions of the lesser ring syndesmosis depending on the mechanism of injury (SER and PER). When the injury is produced, the foot is flat on the floor; internal rotation of the leg and the body relative to the foot results in external talar rotation inside the mortise, and of the fibula. As the talar is displaced by external rotation and eversion inside the mortise, it becomes wider. ${ }^{10}$ Forced dorsiflexion of the ankle introduces the broader part of the talar dome into the mortise, opening the articular space. In all cases, the distal fibula is displaced laterally from its articulation with the distal tibia, ${ }^{14}$ giving rise to a sequential disruption of structures with a predictable pattern: the AITFL (the weakest) and the superficial deltoid ligament are the first to become injured. As the force increases, the IOL and the IOM rupture. The PITFL and the inferior fascicle of the deltoid ligament are the last to completely rupture,

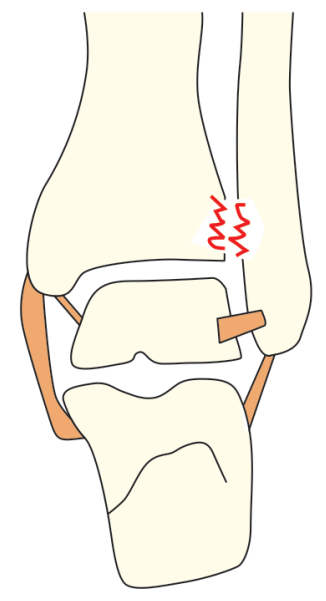

Fig. 4: Supination-external rotation (SER I): the sequence of injury in an SER fracture starts with the disruption of the anterior inferior tibiofibular ligament

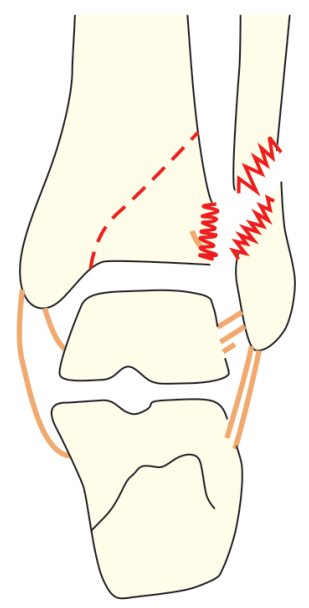

Fig. 6: Supination-external rotation (SER III): the next structure to be affected is the posterior malleolus with the posterior inferior tibiofibular ligament included leading to further instability and/or dislocation of the tibiotalar joint. The severity of the force applied, and its duration determines the proximal extension of the lesion (IOM or proximal fibula).

To determine the spectrum of syndesmotic injuries with the foot in rotation, and to know where and how the three main ligaments are injured, Xu et al. ${ }^{15}$ conducted an imaging study and individually analyzed the different forces and ligaments (AITFL, PITFL, and IOM) involved.

- AITFL: the study ${ }^{15}$ demonstrated increased strain of the proximal band of the AITFL and the midsubstance close to Chaput's tubercle. The fracture usually occurred closer to the fibular site of insertion. The identification of regions of the AITFL with high levels of strain has potential implications for reconstruction and augmentation techniques for this ligament (Fig. 12).

- IOM: the authors showed the IOM to bear oblique strains. This finding is consistent with the anatomical distribution of its fibers, which lie at an oblique angle throughout the IOM. This study does not support the reconstruction of only one ligament to

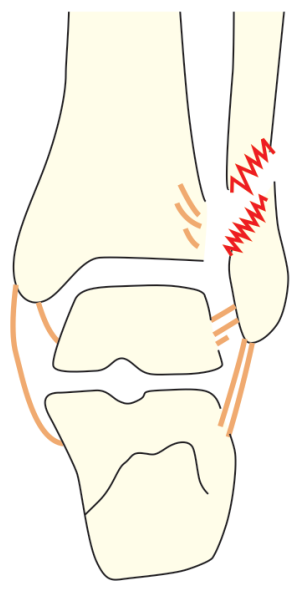

Fig. 5: Supination-external rotation (SER II): the forces continue and provoke an oblique fibula fracture

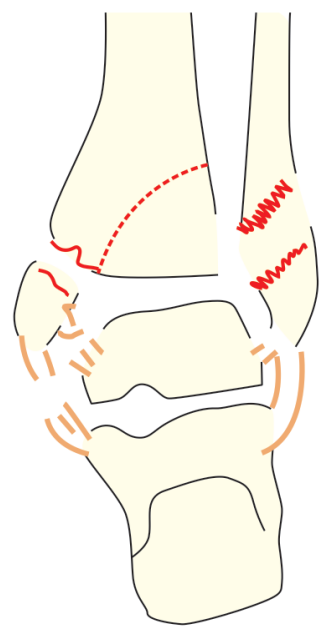

Fig. 7: Supination-external rotation (SER IV): finally, the forces may rupture the deltoid ligament or fracture the medial malleolus 


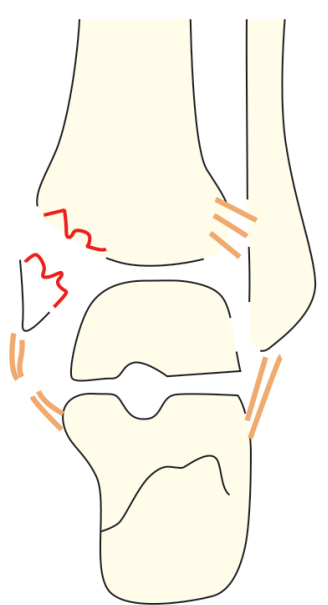

Fig. 8: Pronation-external rotation (PER I): the sequence of injury in a PER fracture starts with the disruption of the deltoid ligament or a medial malleolus transverse fracture

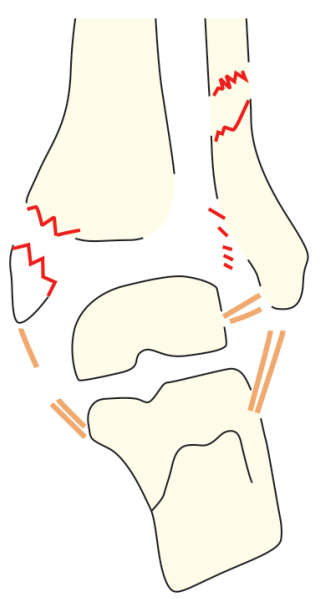

Fig. 10: Pronation-external rotation (PER III): the forces continue and provoke a lateral short oblique or spiral fracture of the fibula (anterosuperior to posteroinferior) above the level of the joint

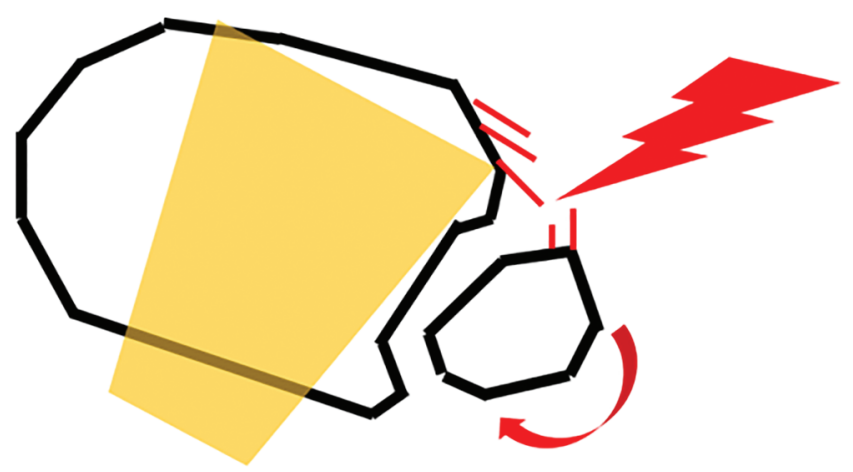

Fig. 12: Anterior inferior tibiofibular ligament (AITFL) pattern injury. The ligament disruption usually takes place in the midsubstance and close to the fibular site of insertion

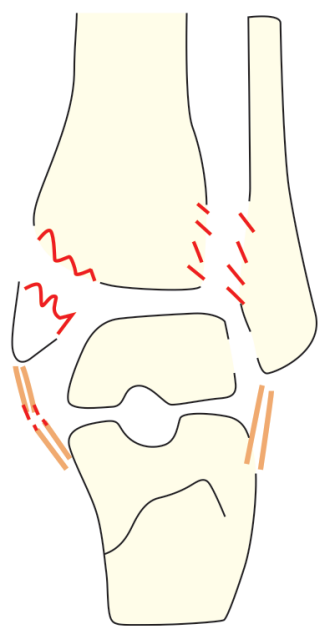

Fig. 9: Pronation-external rotation (PER II): the next structure to be disrupted is the anterior inferior tibiofibular ligament

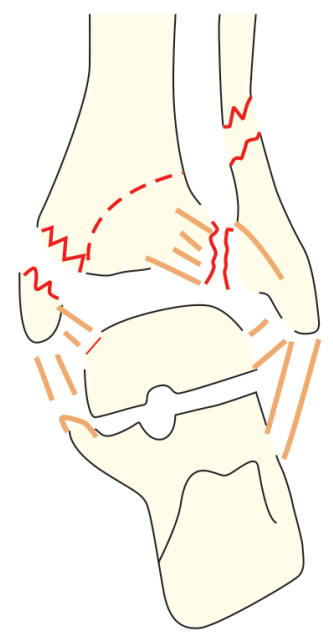

Fig. 11: Pronation-external rotation (PER IV): finally, the forces may provoke an avulsion of the posterior malleolus with the posterior inferior tibiofibular ligament included

completely restore rotational stability in the context of severe ankle fractures in external rotation.

- PITFL: the PITFL is thick and strong and often excessive strain forces ultimately provoke an avulsion fracture of the posterior (lateral) malleolus, rather than a ruptured ligament. ${ }^{16}$ In the study by Warner et al., ${ }^{17}$ which compared magnetic resonance images with surgical findings, 95 to $96 \%$ of PITFL lesions occurred as extensive delaminations of the posterolateral malleolus and not of the midsubstance of the PITFL. When the avulsion fracture of the posterolateral malleolus was reduced, the syndesmosis was also passively reduced and stabilized ("ligamentotaxis") (Fig. 13).

\section{Mechanical Repair}

Anatomical reduction of the tibiofibular distal syndesmosis is essential for the correct tibiotalar kinematics and is vital for the long-term functional outcome of the ankle. ${ }^{18}$ It is important to have a three-dimensional view after injury to this joint, for which surgical 

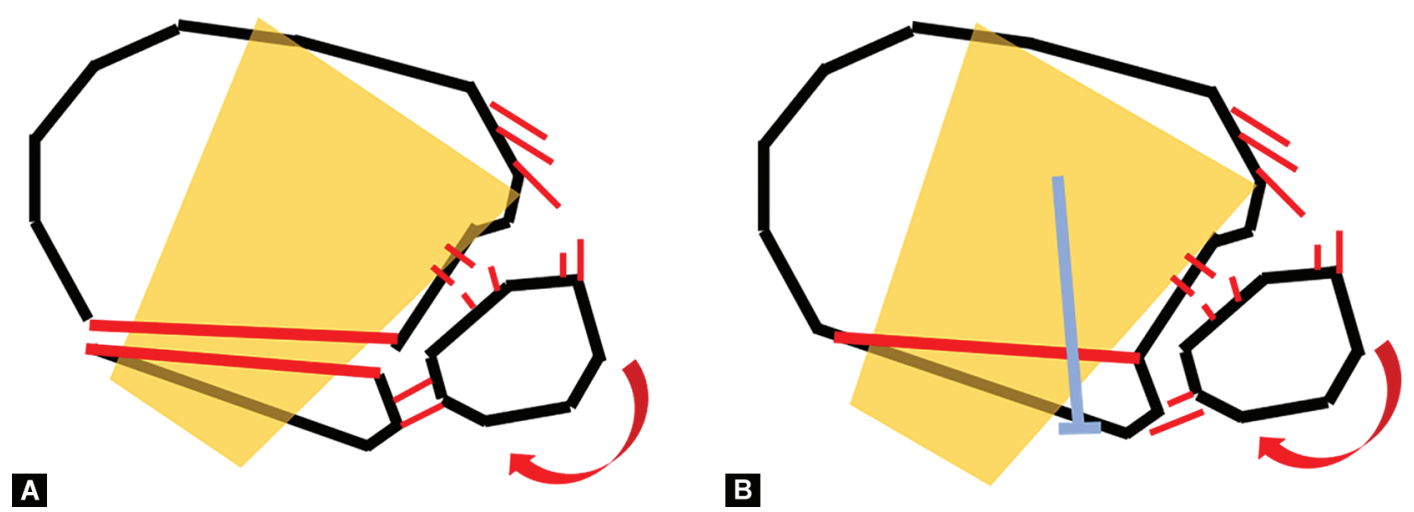

Figs 13A and B: Posterior inferior tibiofibular ligament (PITFL) injury pattern. (A) Strain forces provoke an avulsion fracture of the posterior (lateral) malleolus rather than a ruptured ligament; (B) Fixation of the fracture will reduce the posterior syndesmosis

planning using 3D-computed tomography $(C T)$ imaging is essential. Owing to residual instabilities and secondary degenerative alterations, the classical approach to reducing ankle fractures is now evolving. This change in philosophy entails the reduction and anatomical fixation of each osseous and ligamentous fraction.

The first and most important step in syndesmotic reconstruction is to correctly reduce the lateral malleolus inside the incisura fibularis. The articular surfaces should serve as points of reference. ${ }^{19}$ In the setting of a complete syndesmosis injury, the repair sequence should contemplate direct repair of the lateral and medial malleoli, the posterior edge (insertion of the AITFL), the Chaput-Tillaux or Lefort-Wagstaffe fragment (origin and insertion of the AITFL), and the deltoid ligament. When disconnections are observed without a bone fragment, the ligaments and the avulsed periosteal cuff should be reinserted using suture anchors. ${ }^{20,21}$

Given that the PITFL is the main syndesmotic stabilizer, the importance of the posterior malleolus is being increasingly acknowledged. ${ }^{22-24}$ In the presence of a fracture of the posterior malleolus with a syndesmosis injury, open reduction and fixation are recommended for several reasons, regardless of the size of the bone fragment. First, the posterolateral malleolus includes the posterior edge of the tibial incisura as mentioned previously, and reduction of the syndesmotic components by closed techniques is not very precise. ${ }^{21,23,24}$ By anatomically reducing the posterolateral malleolus, the fibular notch is reconstructed, ensuring a good reduction of the fibula in the tibiofibular joint, with the PITFL recovering its correct position and tension. So joint congruence is much improved and the risk of early post-traumatic ankle arthritis is minimized. ${ }^{23,25}$ The advantages of anatomical repair are unquestionable, as they provide much more precise and stable reductions, without the need to use trans-syndesmotic devices, thus saving on costs and the need for reinterventions to surgically remove them. ${ }^{26}$

From the perspective of surgical mechanical repair and stabilization, two scenarios of injury may be contemplated:

- Syndesmotic ligamentous injury in complex ankle fractures. In this case, the ligaments tend to heal correctly after anatomical reduction of the joint and correct fixation of bone structures. ${ }^{26}$ When a correct bone reduction is achieved, no "extra" syndesmotic fixation system is required.

- Complete syndesmosis injury (affecting the whole syndesmotic ring), with no, or minimal, bone lesion. There is controversy about the optimum point to reach between stable and accommodative fixation. Conceptually, a rigid fixation system does not allow for joint flexibility and produces too much stiffness. By contrast, a more flexible system would help control reduction of the coronal plane but would be insufficient to control loading forces from the sagittal plane and rotational forces acting around the fibula. $8,21,27$

The goals of joint repair are being increasingly orientated toward achieving an "anatomically-aligned mortise", and to reduce the original "stabilization" of the syndesmotic tissues and ligaments to reproduce the original bone kinematics. ${ }^{28}$

\section{New Perspectives on Mechanics of the SYNDESMOSIS}

Research must continue to explore in greater depth the biomechanical and anisotropic behavior of the syndesmotic ligaments. From a clinical perspective, imaging techniques such as weight-bearing CT are now used in patients who can support weight on the foot, to compare these results with those obtained in cadaver studies. ${ }^{21,23,29}$ Similarly, to improve repair techniques, intraoperative $\mathrm{CT}\left(\mathrm{O}\right.$-arm) is being introduced. ${ }^{30,31}$ The aim of this is to confirm, in real-time, a correct reduction of the fibular length and rotation within the fibular notch, the mediolateral displacement, the alignment of the mortise, and the correct positioning of the implants used for fixation.

With regards to the method of syndesmotic fixation, and since the appearance of alternatives to conventional screws, a new line of research opened up focusing on the most suitable stabilization system: suture-button vs syndesmotic screw. ${ }^{21}$ Lee et al. ${ }^{32}$ demonstrated that syndesmotic reduction by fixation with the suture-button results in a less constrained reduction in coronal and sagittal planes compared with screw fixation. Both the configuration of the devices and the number used in both groups had no significant clinical difference. The flexibility offered by the suture button is correlated with positive clinical results in the long term. ${ }^{33}$ However, one must not forget the complications described: swelling of soft tissues, entrapment of the anterior tibial tendon, local infection, and pathological tibial/fibular fractures. ${ }^{32}$ For these reasons, screw fixation continues to be the elective fixation technique in patients with a poorer bone quality, and in those who require special protection of the soft tissues.

The results, combined with laboratory research and clinical outcomes, will, in the future, help us to better understand the 
biomechanics and pathomechanics of syndesmotic ligaments and will lead us to indicate the most suitable fixation method for our patients.

\section{References}

1. Vosseller JT, Karl JW, Greisberg JK. Incidence of syndesmotic injury. Orthopedics 2014;37(3):e226. DOI: 10.3928/01477447-20140225-53.

2. Hunt KJ, George E, Harris AH, et al. Epidemiology of syndesmosis injuries in intercollegiate football: incidence and risk factors from National collegiate athletic association injury surveillance system data 2004-2005 to 2008-2009. Clin J Sport Med 2013;23(4):278-282. DOI: 10.1097/JSM.0b013e31827ee829.

3. Waterman BR, Belmont PJ, Cameron KL, et al. Risk factors for syndesmotic and medial ankle sprain: role of sex, sport, and level of competition. Am J Sports Med 2011;39(5):992-998. DOI: $10.1177 / 0363546510391462$.

4. https://www.ncbi.nlm.nih.gov/pubmed.

5. Hermans JJ, Beumer A, de Jong TAW, et al. Anatomy of the distal tibiofibular syndesmosis in adults: a pictorial essay with multimodality approach. J Anat 2010;217(6):633-645. DOI: 10.1111/j.1469-7580.2010.01302.x.

6. Perry J. Gait analysis: normal and pathological function. Thorofare (New Jersey): Slack 1992;12(6):815. DOI: 10.1097/01241398-19921100000023.

7. Ogilvie-Harris DJ, Reed SC, Hedman TP. Disruption of the ankle syndesmosis: biomechanical study of the ligamentous restraints. Arthroscopy 1994;10(5):558-560. DOI: 10.1016/s0749-8063(05)800143.

8. Clanton TO, Williams BT, Backus JD, et al. Biomechanical analysis of the individual ligament contributions to syndesmotic stability. Foot Ankle Int 2017;38(1):66-75. DOI: 10.1177/1071100716666277.

9. Kelikian H, Kelikian S. Disorders of the ankle. Philadelphia: W.B. Saunders Company; 1985 . pp. 4-8.

10. Hunt KJ, Goeb Y, Behn AW, et al. Ankle joint contact load and displacement with progressive syndesmotic injury. Foot Ankle Int 2015;36(9):1095-1103. DOI: 10.1177/1071100715583456.

11. Harris J, Fallat L. Effects of isolated Weber B fibular fractures on the tibiotalar contact area. J Foot Ankle Surg 2004;43(1):3-9. DOI: 10.1053/j.jas.2003.11.008.

12. Ramsey PL, Hamilton W. Changes in tibiotalar area of contact caused by lateral talar shift. J Bone Joint Surg 1976;58-A(3):356-357. DOI: 10.2106/00004623-197658030-00010.

13. Doughtie M. Syndesmotic ankle sprains in football: a survey of national football league athletic trainers. J Athl Train 1999;34(1):15-18.

14. Haraguchi N, Armiger RS. A new interpretation of the mechanism of ankle fracture. J Bone Joint Surg Am 2009;91(4):821-829. DOI: 10.2106/ JBJS.G.01288.

15. $X u$ D, Wang $Y$, Jiang $C$, et al. Strain distribution in the anterior inferior tibiofibular ligament, posterior inferior tibiofibular ligament, and interosseous membrane using digital image correlation. Foot Ankle Int 2018;39(5):618-628. DOI: 10.1177/1071100717753160.

16. Van de Perre $S$, Vanhoenacker FM, De Vuyst $D$, et al. Imaging anatomy of the ankle. JBR-BTR 2004;87(6):310-314.

17. Warner SJ, Garner MR, Schottel PC, et al. Analysis of PITFL injuries in rotationally unstable ankle fractures. Foot Ankle Int 2015;36(4):377382. DOI: $10.1177 / 1071100714558845$.
18. Sagi HC, Shah AR, Sanders RW. The functional consequence of syndesmotic joint malreduction at a minimum 2-year follow-up. J Orthop Trauma 2012;26(7):439-443. DOI: 10.1097/ BOT.0b013e31822a526a.

19. Bartonícek J. Anatomy of the tibiofibular syndesmosis and its clinical relevance. Surg Radiol Anat 2003;25(5-6):379-386. DOI: 10.1007/ s00276-003-0156-4.

20. Fong Mak M, Stern R, Assal M. Repair of syndesmosis injury in ankle fractures: current state of the art. EOR 2018;3:24-29.

21. Clanton TO, Whitlow SR, Williams BT, et al. Biomechanical comparison of 3 current ankle syndesmosis repair techniques. Foot Ankle Int 2017;38(2):200-207. DOI: 10.1177/1071100716666278.

22. Miller MA, McDonald TC, Graves ML, et al. Stability of the syndesmosis after posterior malleolar fracture fixation. Foot Ankle Int 2018;39(1):99-104. DOI: 10.1177/1071100717735839.

23. Bartoníček J, Rammelt S, Tuček M. Posterior malleolar fractures: changing concepts and recent developments. Foot Ankle Clin 2017;22(1):125-145. DOI: 10.1016/j.fcl.2016.09.009.

24. Vacas-Sánchez E, Olaya-González C, Abarquero-Diezhandino A, et al. How to address the posterior malleolus in ankle fractures? A decisionmaking model based on the computerised tomography findings. Int Orthop 2020;44(6):1177-1185. DOI: 10.1007/s00264-020-04481-5.

25. Abarquero-Diezhandino A, Luengo-Alonso G, Alonso-Tejero D, et al. Study of the relation between the posterior malleolus fracture and the development of osteoarthritis. Rev Esp Cir Ortop Traumatol 2020;64(1):41-49. DOI: 10.1016/j.recote.2019.11.002.

26. Schottel PC, Baxter J, Gilbert S, et al. Anatomic ligament repair restores ankle and syndesmotic rotational stability as much as syndesmotic screw fixation. J Orthop Trauma 2016;30(2):e36-e40. DOI: 10.1097/ BOT.0000000000000427.

27. La Mothe JM, Baxter JR, Murphy C, et al. Three-dimensional analysis of fibular motion after fixation of syndesmotic injuries with a screw or suturebutton construct. Foot Ankle Int 2016;37(12):1350-1356. DOI: 10.1177/1071100716666865.

28. Kwon JY, Cronin P, Velasco B, et al. Evaluation and significance of mortise instability in supination external rotation fibula fractures: a review article. Foot Ankle Int 2018;39(7):865-873. DOI: 10.1177/1071100718768509.

29. Malhotra K, Welck M, Cullen N, et al. The effects of weight bearing on the distal tibiofibular syndesmosis: a study comparing weight bearing-CT with conventional CT. Foot Ankle Surg 2018(4). DOI: 10.1016/j.fas.2018.03.006.

30. Hsu AR, Gross CE, Lee S. Intraoperative O-arm computed tomography evaluation of syndesmotic reduction: case report. Foot Ankle Int 2013;34(5):753-759. DOI: 10.1177/1071100712468872.

31. Lintz F, Netto C, Barg A, et al. Weight-bearing cone beam CT scans in the foot and ankle. EFORT Open Rev 2018;3(5):278-286. DOI: 10.1302/2058-5241.3.170066.

32. Lee JS, Curnutte B, Pan K, et al. Biomechanical comparison of suturebutton, bioabsorbable screw, and metal screw for ankle syndesmotic repair: a meta-analysis. Foot Ankle Surg 2021;27(2):117-122. DOI: 10.1016/j.fas.2020.03.008.

33. Grassi A, Samuelsson K, D'Hooghe P, et al. Dynamic stabilization of syndesmosis injuries reduces complications and reoperations as compared with screw fixation: a meta-analysis of randomized controlled trials. Am J Sports Med 2020;48(4):1000-1013. DOI: 10.1177/0363546519849909. 